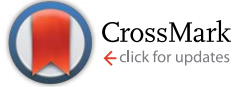

Cite this: Soft Matter, 2015, 11, 600

\title{
Temperature and pressure tuneable swollen bicontinuous cubic phases approaching nature's length scales $\dagger$
}

\author{
H. M. G. Barriga, A. I. I. Tyler, N. L. C. McCarthy, E. S. Parsons, O. Ces, R. V. Law, \\ J. M. Seddon* and N. J. Brooks*
}

Bicontinuous cubic structures offer enormous potential in applications ranging from protein crystallisation to drug delivery systems and have been observed in cellular membrane structures. One of the current bottlenecks in understanding and exploiting these structures is that cubic scaffolds produced in vitro are considerably smaller in size than those observed in biological systems, differing by almost an order of magnitude in some cases. We have addressed this technological bottleneck and developed a methodology capable of manufacturing highly swollen bicontinuous cubic membranes with length scales approaching those seen in vivo. Crucially, these cubic systems do not require the presence of proteins. We have generated highly swollen $1 \mathrm{~m} 3 \mathrm{~m}$ symmetry bicontinuous cubic phases with lattice parameters of up to $480 \AA$, composed of ternary mixtures of monoolein, cholesterol and negatively charged lipid (DOPS or DOPG) and we have been able to tune their lattice parameters. The swollen cubic phases are highly sensitive to both temperature and pressure; these structural changes are likely to be controlled by a fine balance between lipid headgroup repulsions and lateral pressure in the hydrocarbon chain region.

Received 24th October 2014 Accepted 18th November 2014

DOI: $10.1039 / c 4 s m 02343 a$

www.rsc.org/softmatter
Lipids exhibit rich polymorphism in water, forming a range of lyotropic mesophases. These mesophases range in structure from the simple lamellar planar bilayers to highly curved structures such as the inverse hexagonal $\left(\mathrm{H}_{\mathrm{II}}\right)$ and cubic phases. Cubic phases are divided into two categories; bicontinuous and micellar. The inverse bicontinuous cubic phases have a continuous fluid lipid bilayer, separating two interwoven continuous water networks. The continuous fluid lipid bilayer is draped over an infinite periodic minimal surface (IPMS) and the symmetry is defined by its space group. There are 36 theoretical cubic space groups, however only a small number have so far been observed in lipid systems. ${ }^{7}$ Inverse bicontinuous cubic phases have been observed with gyroid $\left(\mathrm{Q}_{\mathrm{II}}^{\mathrm{G}}\right)$, double diamond $\left(\mathrm{Q}_{\text {II }}{ }^{\mathrm{D}}\right)$ and primitive $\left(\mathrm{Q}_{\text {II }}{ }^{\mathrm{P}}\right)$ architecture corresponding to space groups $I a 3 d$, Pn $3 m$ and $I m 3 m$ respectively. Examples of these are shown in Fig. 1.

Inverse bicontinuous cubic phases have been observed in vivo in the organised smooth endoplasmic reticulum, ${ }^{8}$ as well as in amoeba mitochondria, ${ }^{9}$ with lattice parameters on the order of $1000 \AA$ A. However, to date few in vitro models have been generated with lattice parameters approaching those seen in vivo. ${ }^{5,10-12}$ Previous calculations by Bruinsma ${ }^{13}$ suggested that there is an upper limit on bicontinuous cubic phase lattice parameters of approximately $300 \AA$, above which thermal fluctuations would destroy the ordering and lead to the breakdown of the cubic structure. However, our work shows that by incorporating charged lipids and cholesterol into bicontinuous cubic 


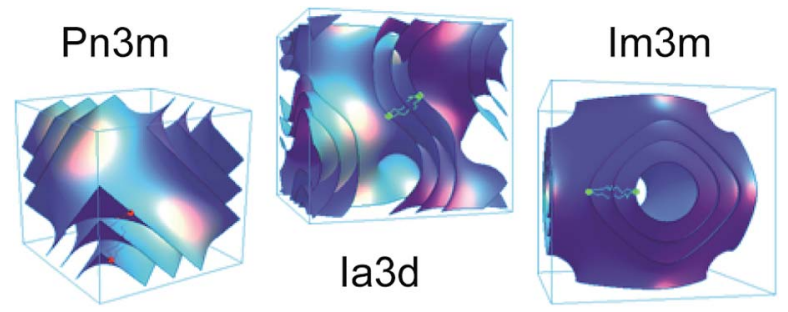

Fig. 1 The observed inverse bicontinuous cubic phases displaying double diamond $\left(Q_{\|}{ }^{D}\right)$, gyroid $\left(Q_{\|}{ }^{G}\right)$, or primitive $\left(Q_{\|}{ }^{P}\right)$ architecture corresponding to space groups $P n 3 m, 1 a 3 d$, and $I m 3 m$ respectively.

phases, it is possible to stabilise these structures and surpass this limit. Cholesterol is a major component of cell membranes ${ }^{14}$ reaching up to $50 \mathrm{~mol} \%$ of the total lipid composition. It has also been shown to significantly increase the bending rigidity ${ }^{15}$ of bilayers and when added to monoolein has enabled the crystallisation of many GPCRs. ${ }^{16}$ Although its addition to monoolein has previously only resulted in a marginal increase in lattice parameter, its ability to modulate membrane mechanics and significance for future protein incorporation applications makes it an essential component of our experiments. ${ }^{12}$

As mentioned above, engineering swollen bicontinuous cubic phases is of great interest for membrane protein crystallisation. Cubic phases have been successfully used to crystallise and deliver high resolution structures of human GPCRs and photosynthetic proteins. ${ }^{3,4}$ A key factor in successful in cubo crystallisation is maintaining the ability of the proteins to move laterally within the bilayer, so allowing effective nucleation and crystal growth. The limit on accessible length scales therefore significantly limits the range of proteins to which this technique can be applied. ${ }^{17}$

Monoglycerides are the archetypal bicontinuous cubic phase forming lipids and generally exhibit lattice parameters of less than $100 \AA$ in excess water. Different approaches have been used to extend the limits of the lattice parameters available in these systems, including the addition of hydration modulating surfactants such as octylglucoside (OG), ${ }^{17,18}$ sugars (sucrose stearate) ${ }^{19}$ and phospholipids. ${ }^{12,20}$ Octylglucoside (10 mol\%) added to monolinolein (hydrated with $57 \mathrm{wt} \%$ water) is known to adopt a $P n 3 m$ structure with a lattice parameter of $128 \AA$ at $20{ }^{\circ} \mathrm{C}$ (ref. 17) and $153 \AA$ when added to monoolein (in excess phosphate buffer) at the same concentration and temperature. ${ }^{18}$ Sucrose stearate $(20 \mathrm{~mol} \%)$ added to a commercial grade monoglyceride mixture (hydrated to $52 \mathrm{wt} \%$ with phosphate buffer) gives a Pn $3 m$ lattice parameter of approximately $150 \AA$ at $37{ }^{\circ} \mathrm{C} .{ }^{19}$ Some pure phospholipids are also known to form bicontinuous cubic phases and the kinetics of non-lamellar phase formation in $\mathrm{N}$-monomethyl-dioleoyl phosphatidylethanolamine (DOPE-Me) has been investigated as a model for biomembrane fusion. ${ }^{21,22}$ Two main pathways for cubic phase formation from a lamellar phase were reported, the first from constant temperature incubation at $55{ }^{\circ} \mathrm{C}$ yielding an $\mathrm{Im} 3 \mathrm{~m}$ lattice parameter of $330 \AA$ and the second through an indirect conversion in a heating cooling cycle yielding an $\operatorname{Im} 3 \mathrm{~m}$ lattice parameter of $403 \AA$. It should be noted that there are significant kinetic barriers to overcome in these systems which would be likely to make active tuning of the cubic phase impractical. Addition of charged dioleoylphosphatidylserine (DOPS) ${ }^{12}$ and distearoyl phosphatidylglycerol (DSPG) ${ }^{20}$ to monoolein has also been shown previously to induce swelling in cubic phases; 8 mol\% DOPS in monoolein at $4{ }^{\circ} \mathrm{C}(60 \mathrm{wt} \%$ water $)$ forms an $\mathrm{Im} 3 \mathrm{~m}$ phase with a lattice parameter of $255 \AA$ and 3 mol\% DSPG in monoolein at $22{ }^{\circ} \mathrm{C}(70 \mathrm{wt} \%$ water $)$ exhibits an $\operatorname{Im} 3 m$ cubic phase with a lattice parameter of $268 \AA$. To the best of our knowledge, these examples show the largest lattice parameters found to date in pure lipid systems.

Recent work has highlighted the bio-technical use of monoglyceride based bicontinuous cubic phases. Incorporation of double stranded siRNA molecules into bicontinuous cubic phases has enabled delivery of sequence-specific mRNA with virtually no negative implications for the cell. ${ }^{10}$ It was also noted in this work that addition of the cationic lipid 1,2-dioleoyl-3trimethylammonium-propane (DOTAP) caused the bicontinuous cubic phase to swell up to a lattice parameter of $220 \AA .{ }^{10}$ In a separate study, the activity and kinetics of horseradish peroxidase (HRP) contained within the pores of a bicontinuous cubic phase were measured as a function of water channel size. The addition of the enzyme and sucrose stearate swelled the lipid structure's water channel diameter and HRP showed increased activity in a more highly swollen cubic phase. ${ }^{19}$

It should be noted that some block copolymers are capable of forming bicontinuous cubic phases, which can be extremely stable and can have very large lattice parameters (up to $500 \AA$ ). ${ }^{23}$ However in contrast to lipid based structures, these are often not biocompatible and do not provide good biomembrane mimics.

We have used temperature and pressure to modulate the lattice parameters of highly swollen lipidic cubic phases formed from monoolein (MO), cholesterol (chol) and the charged lipids 1,2-dioleoyl-sn-glycero-3-phospho(1'-rac-glycerol) (DOPG) and 1,2-dioleoyl-sn-glycero-3-phospho-L-serine (DOPS). These structures, which display lattice parameters of almost $500 \AA$, have allowed us to investigate the parameters that enable lattice expansion and begin to actively control the lattice size. By understanding how to swell and control the behaviour of pure bicontinuous cubic phases we aim to make significant steps towards designing active membrane structures that can be specifically targeted to bio-technical applications.

\section{Experimental details}

\section{Materials}

1,2-Dioleoyl-sn-glycero-3-phospho(1'-rac-glycerol) (sodium salt) (DOPG), 1,2-dioleoyl-sn-glycero-3-phospho-L-serine (sodium salt) (DOPS) and cholesterol (ovine, wool) (chol) were purchased from Avanti Polar Lipids (AL, USA) as lyophilised powders. 1Oleoyl-rac-glycerol (monoolein, MO) was purchased from Sigma Aldrich (Gillingham, UK). The lipids had a purity of $>98 \%$ and were used without further purification, but were lyophilised for 12 hours before use to ensure they were fully dry. 


\section{Methods}

Lipid mixtures were prepared by co-dissolving appropriate masses of the lipids in chloroform (Sigma Aldrich, Gillingham, UK). These were then dried under a stream of nitrogen gas for 3 hours and then under vacuum for a minimum of 12 hours, after which they were sealed and stored at $-20{ }^{\circ} \mathrm{C}$ before use. Samples were hydrated in HPLC grade water (VWR, UK) to a minimum of $70 \mathrm{wt} \%$ water. After hydration, each sample was heat cycled (between approximately $-200{ }^{\circ} \mathrm{C}$ and $60{ }^{\circ} \mathrm{C}$ ) a minimum of twenty times. Small angle X-ray diffraction (SAXS) data were obtained at Diamond Light Source using beamline I22 and at the European Synchrotron Radiation Facility (ESRF) using beamline ID02. Diffraction patterns were collected using an X-ray wavelength of $0.73 \AA$ (ESRF) or $0.69 \AA$ (Diamond Light Source) and at camera lengths of $1.2 \mathrm{~m}, 3.0 \mathrm{~m}, 4.0 \mathrm{~m}$ and $4.5 \mathrm{~m}$. Samples were contained in Teflon sample holders with mylar windows and then pressurised using a custom built high pressure cell. ${ }^{24}$ The structural behaviour of the highly swollen cubic structures was mapped between $25-65{ }^{\circ} \mathrm{C}$ and 0-200 MPa.

For some samples, pressure caused the collapse of the cubic phase to a lamellar or less swollen cubic phase. All data presented here are for cubic phases showing stability over the temperature and pressure range studied. Pressure-temperature analysis was performed on binary and ternary mixtures which showed stable behaviour with a high concentration of charged lipid. Molar ratios were selected based on them showing stable cubic phase formation in preliminary experiments. For MO : chol : DOPG mixtures, molar ratios of $95: 0: 5,91: 5: 4$ and $80: 15: 5$ were investigated whilst for MO:chol:DOPS mixtures, molar ratios of $90: 0: 10,85: 5: 10,75: 15: 10$ and $65: 30: 5$ were selected. Highly swollen cubic phases were found between 45 and $55{ }^{\circ} \mathrm{C}$ for the DOPG ternary mixtures and between 25 and $65{ }^{\circ} \mathrm{C}$ for the DOPS ternary mixtures.

\section{Results}

Lipid mixtures that were found to form highly swollen bicontinuous cubic phases were investigated as a function of temperature, pressure and composition. All mixtures studied adopted Im $3 m$ symmetry bicontinuous cubic phases with lattice parameters ranging from 290 to $480 \AA$ (an example characteristic Im $3 m$ diffraction pattern is shown in Fig. 2). To decouple the effects of the charged lipids within the ternary mixture, binary mixtures of MO with DOPS-DOPG were first investigated, followed by ternary mixtures which also included cholesterol.

It should be noted that while in some cases, addition of cholesterol led to a small reduction in the lattice parameter, cholesterol was required to achieve the highest lattice parameter observed in these experiments. Cholesterol also significantly increases the sensitivity of the lattice parameter to pressure, facilitating structural tuning, and will be required for future protein incorporation applications.

\section{Effect of temperature and pressure on binary mixtures}

The MO : DOPS $90: 10 \mathrm{~mol} \%$ binary mixture adopts a highly swollen bicontinuous cubic $\operatorname{Im} 3 m$ structure. The effect of

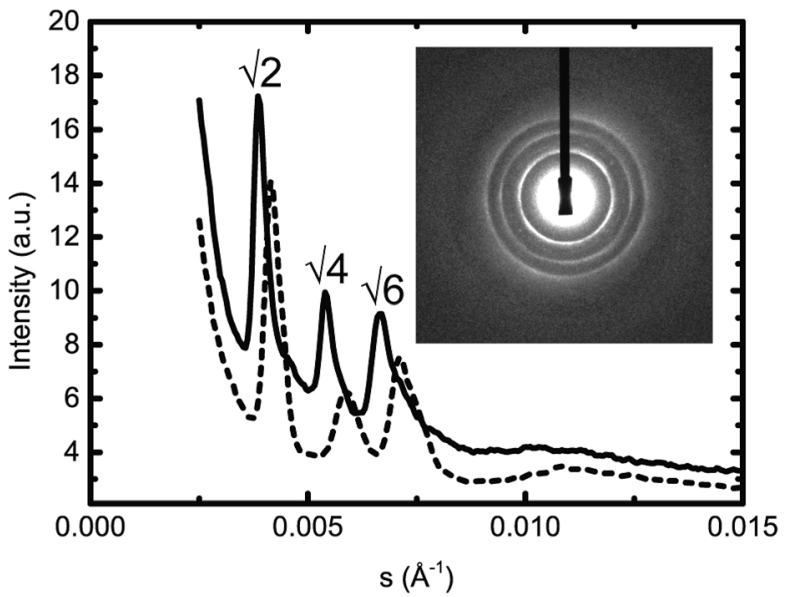

Fig. 2 Integrated diffraction patterns from the highly swollen $1 \mathrm{~m} 3 \mathrm{~m}$ bicontinuous cubic phase adopted by MO : chol: DOPG $80: 15: 5$ mol\% at $45{ }^{\circ} \mathrm{C}$, at atmospheric pressure (solid line) and $200 \mathrm{MPa}$ (dashed line). Insert: corresponding 2D diffraction pattern at atmospheric pressure.

pressure and temperature on its lattice parameter is shown in Fig. 3. This reaches a maximum of $400 \AA$ at $45{ }^{\circ} \mathrm{C}$ and atmospheric pressure, which is $180 \AA$ larger than the maximum lattice parameter previously reported for MO : DOPS mixtures. ${ }^{\mathbf{1 2}}$ This difference is likely to be due to hydration of the lipid mixture; the experiments described here have used $70 \mathrm{wt} \%$ water, whereas the previously reported $220 \AA$ lattice parameter was obtained with $60 \mathrm{wt} \%$ water. This implies that the maximum hydration of these mixtures is above $60 \mathrm{wt} \%$ and is discussed further later.

Increasing the temperature from 25 to $45^{\circ} \mathrm{C}$ at atmospheric pressure causes the lattice parameter of this mixture to increase by approximately $100 \AA$ (5.0 $\mathrm{A}$ per $\left.{ }^{\circ} \mathrm{C}\right)$. In contrast, increasing the pressure from atmospheric pressure to $200 \mathrm{MPa}$ has a relatively small effect, causing a decrease in the lattice

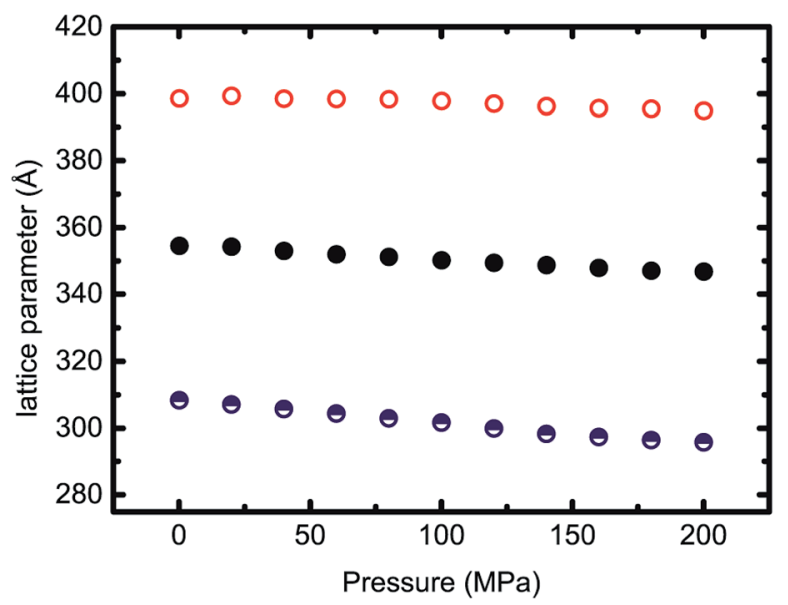

Fig. 3 Effects of temperature and pressure on the lattice parameter of a swollen $1 \mathrm{~m} 3 \mathrm{~m}$ bicontinuous cubic phase composed of MO : DOPS 90 : 10 mol\% shown at $25{ }^{\circ} \mathrm{C}(\bullet), 35^{\circ} \mathrm{C}(\bullet), 45^{\circ} \mathrm{C}(0)$. Error bars are approximately the sizes of the data points. 
parameter of $4 \AA$ at $45^{\circ} \mathrm{C}(0.02 \AA$ per MPa $)$ and $15 \AA$ at $25{ }^{\circ} \mathrm{C}$ $(0.08 \AA$ per MPa). The temperature response found here differs from that previously reported for MO : DOPS between 4 and $20{ }^{\circ} \mathrm{C}$ (where a small decrease is observed)..$^{12}$ Below approximately $20^{\circ} \mathrm{C}$ pure $\mathrm{MO}$ is known to exist as coexisting lamellar crystalline and $P n 3 m$ phases,$^{25}$ this change in the structural behaviour of MO is likely to account for the different behaviour seen here.

Binary mixtures of MO:DOPG 95:5 mol\% showed a maximum lattice parameter of $405 \AA$ at $55^{\circ} \mathrm{C}$ (ESI Fig. S1 $\dagger$ ) and a $40 \AA$ increase in lattice parameter with a temperature increase from $35{ }^{\circ} \mathrm{C}$ to $55{ }^{\circ} \mathrm{C}\left(2.0 \AA\right.$ per $\left.{ }^{\circ} \mathrm{C}\right)$. Increasing the pressure from $0 \mathrm{MPa}$ to $200 \mathrm{MPa}$ led to a relatively small decrease in lattice parameter at $35{ }^{\circ} \mathrm{C}(0.04 \AA$ per MPa $)$ and a small increase in lattice parameter at $45{ }^{\circ} \mathrm{C}(0.05 \AA$ per MPa $)$ and $55^{\circ} \mathrm{C}(0.04 \AA$ per MPa).

\section{Effects of temperature and pressure on ternary mixtures}

The effect of pressure and temperature on the lattice parameter of ternary mixtures of MO:chol:DOPG and MO : chol : DOPS are shown in Fig. 4 and 5. All of the mixtures studied showed highly swollen bicontinuous cubic phases with space group $\operatorname{Im} 3 \mathrm{~m}$ and lattice parameters ranging from $300 \AA$ (MO : chol : DOPG $91: 5: 4,55{ }^{\circ} \mathrm{C}, 200 \mathrm{MPa}$ ) to $480 \AA$ (MO : chol : DOPS $60: 30: 5,54{ }^{\circ} \mathrm{C}$, atmospheric pressure).

Ternary mixtures of MO : chol : DOPG with molar ratios of $80: 15: 5$ and $91: 5: 4$ showed a significant decrease in lattice parameter both with increasing temperature and increasing pressure as shown in Fig. 4. This is notable as generally in lipid systems, the effects of increasing temperature and increasing pressure tend to oppose each other. ${ }^{26}$ For the sample with $5 \mathrm{~mol} \%$ DOPG and $15 \mathrm{~mol} \%$ chol, the lattice parameter decreases by $55 \AA$ between 45 and $55{ }^{\circ} \mathrm{C}\left(5.5 \AA\right.$ per $\left.{ }^{\circ} \mathrm{C}\right)$ and decreases by approximately $25 \AA$ between atmospheric pressure and $200 \mathrm{MPa}(0.13 \AA$ per MPa). This change is smaller for the sample with $4 \mathrm{~mol} \%$ DOPG and $5 \mathrm{~mol} \% \mathrm{chol}$; the lattice parameter decreases by $40 \AA$ between 45 and $55^{\circ} \mathrm{C}\left(4.0 \AA\right.$ per $\left.{ }^{\circ} \mathrm{C}\right)$ and decreases by $20 \AA$ between atmospheric pressure and 200 $\mathrm{MPa}(0.10 \AA$ A per MPa). It should be noted that this behaviour is different to that of the binary mixtures, which show an increase in lattice parameter with increasing temperature.

The ternary mixtures of MO : chol : DOPS at molar ratios of $65: 30: 5$ and $85: 5: 10$ showed an increase in lattice parameter with increasing temperature and a decrease in lattice parameter with increasing pressure as seen in Fig. 5. Similar responses to pressure and temperature were seen for a ternary sample containing MO : chol : DOPS $75: 15: 10 \mathrm{~mol} \%$ (see ESI Fig. S2 $\dagger$ ).

For the sample containing $10 \mathrm{~mol} \%$ DOPS and $5 \mathrm{~mol} \% \mathrm{chol}$, the lattice parameter increases by $25 \AA$ between 26 and $65{ }^{\circ} \mathrm{C}$ $\left(0.6 \AA\right.$ per $\left.{ }^{\circ} \mathrm{C}\right)$ and decreases by $10 \AA$ between atmospheric pressure and $200 \mathrm{MPa}(0.05 \AA$ per MPa). The decrease in lattice parameter with increasing pressure was consistent across different temperatures.
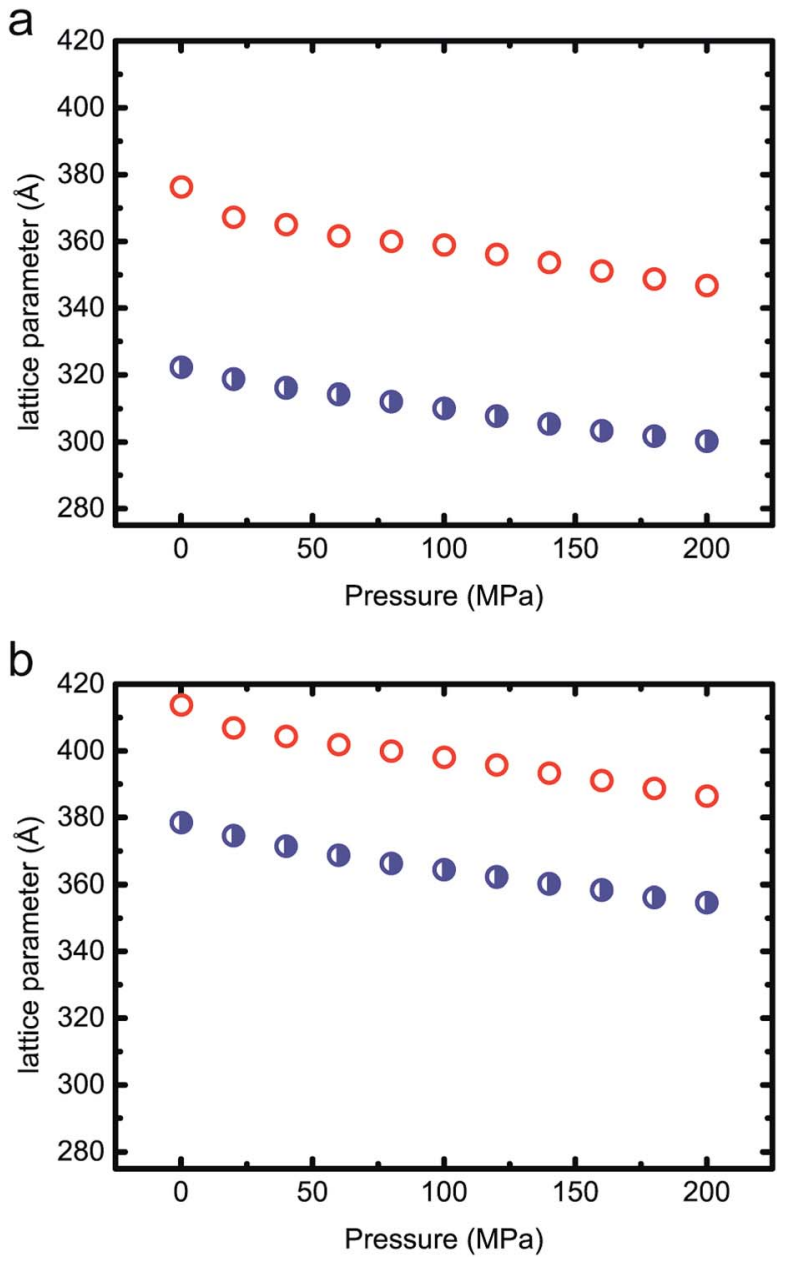

Fig. 4 Effects of temperature and pressure on the lattice parameter of a swollen bicontinuous cubic phase $1 \mathrm{~m} 3 \mathrm{~m}$ composed of (a) $\mathrm{MO}$ : chol: DOPG 80 : $15: 5$ mol\% (b) MO : chol: DOPG $91: 5: 4$ mol\% shown at $45^{\circ} \mathrm{C}(0), 55^{\circ} \mathrm{C}(\mathrm{O})$. Error bars are approximately the sizes of the data points.

For the sample containing $5 \mathrm{~mol} \%$ DOPS and $30 \mathrm{~mol} \% \mathrm{chol}$, the lattice parameter increases by $40 \AA$ between 26 and $54{ }^{\circ} \mathrm{C}$ $\left(1.4 \AA\right.$ per $\left.{ }^{\circ} \mathrm{C}\right)$ and decreases by $12 \AA$ between atmospheric pressure and $200 \mathrm{MPa}$. It should be noted that excess cholesterol crystals were evident in this sample (cholesterol crystals exhibit a characteristic, sharp reflection in the diffraction pattern). Above $45^{\circ} \mathrm{C}$, a hexagonal phase was found to coexist with the swollen $\operatorname{Im} 3 \mathrm{~m}$, but was removed by increasing pressure (the pressure required to remove the hexagonal phase was dependent on temperature as expected). While the hydrolysis of MO in excess water to form glycerol and oleic acid has been shown to promote hexagonal phase formation, ${ }^{27}$ the consistent disappearance of the $\mathrm{H}_{\text {II }}$ phase with pressure (Fig. 6) suggests that the presence of the $\mathrm{H}_{\text {II }}$ phase is not due to hydrolysis in this case but instead a stable structure for this mixture. The positive phase boundary gradient between the $\operatorname{Im} 3 m$ and $\operatorname{Im} 3 m-\mathrm{H}_{\text {II }}$ coexistence region (Fig. 6), demonstrates that despite the complex behaviour of the lattice parameter with temperature and pressure, the phase transition varies as expected. ${ }^{28,29}$ 

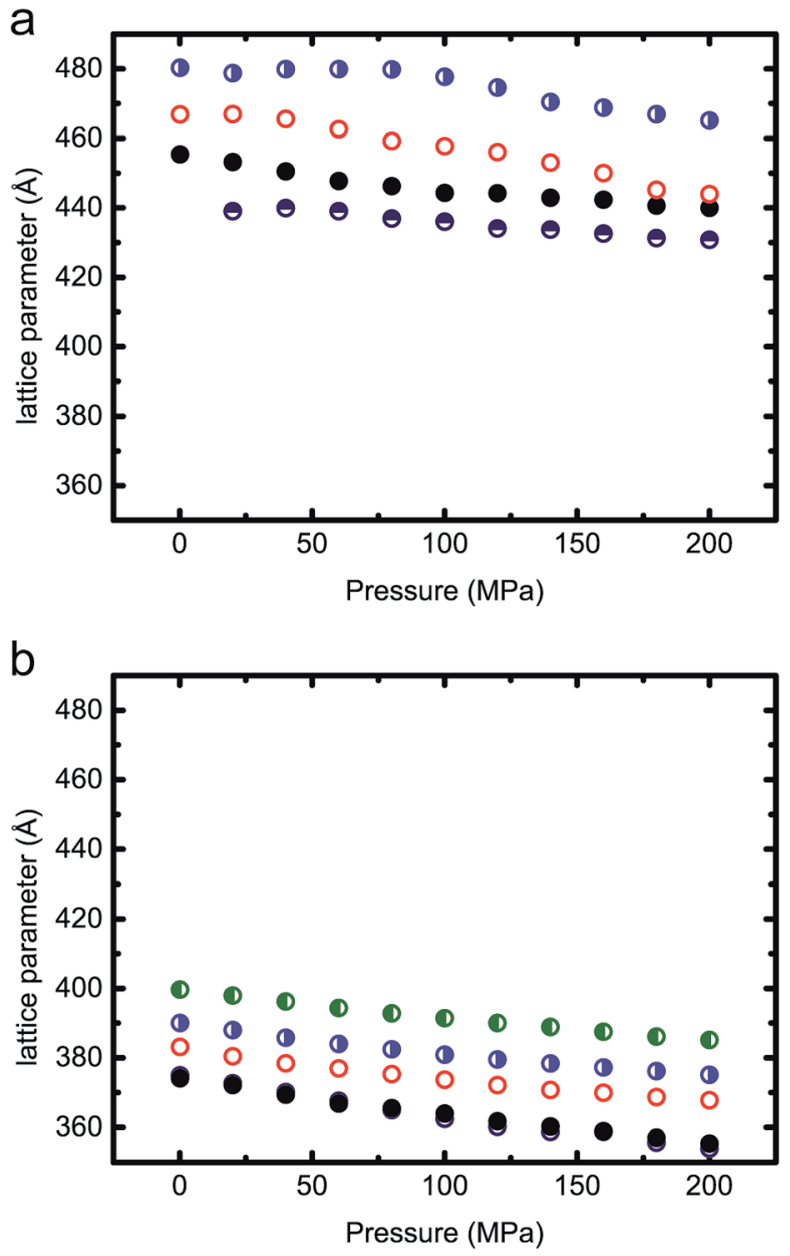

Fig. 5 Effects of temperature and pressure on the lattice parameter of a swollen bicontinuous cubic phase $\operatorname{lm} 3 \mathrm{~m}$ composed of (a) MO : chol : DOPS 65 : $30: 5 \mathrm{~mol} \%$ at $26^{\circ} \mathrm{C}(\bullet), 35^{\circ} \mathrm{C}(\bullet), 45^{\circ} \mathrm{C}(0), 54^{\circ} \mathrm{C}(\bullet)$ (b) $\mathrm{MO}$ : chol : DOPS 85 : 5 : $10 \mathrm{~mol} \%$ shown at $26{ }^{\circ} \mathrm{C}(\bullet), 35^{\circ} \mathrm{C}(\bullet)$, $46^{\circ} \mathrm{C}(0), 55^{\circ} \mathrm{C}(\mathbf{(}), 65^{\circ} \mathrm{C}(\circlearrowleft)$. Error bars are approximately the sizes of the data points.

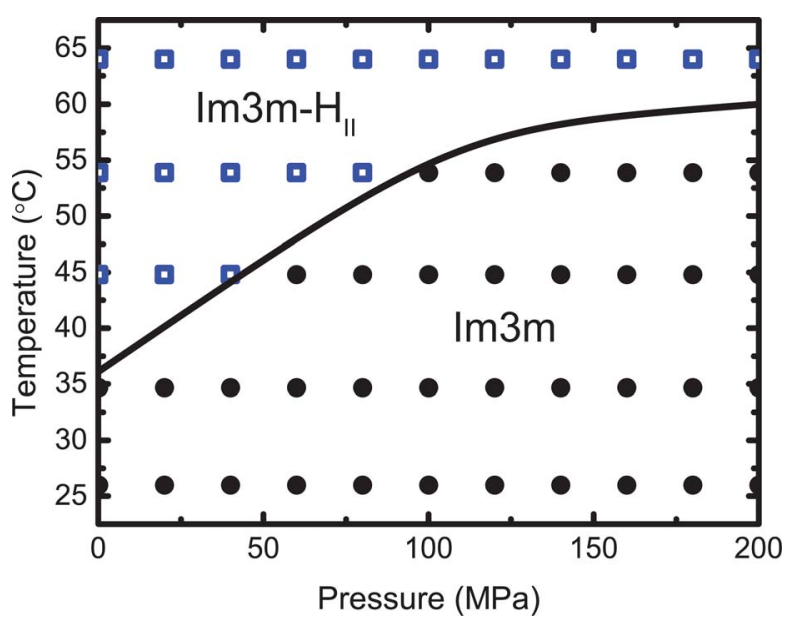

Fig. 6 Pressure-temperature phase behaviour of MO : chol: DOPS $65: 30: 5 \mathrm{~mol} \%$, showing the phase coexistence of $\operatorname{Im} 3 \mathrm{~m}$ with $\mathrm{H}_{\|}$ from approximately $36^{\circ} \mathrm{C}$ to $65^{\circ} \mathrm{C}$, at atmospheric pressure.

\section{Discussion}

The highly swollen cubic structures reported here, with lattice parameters of up to $480 \AA$, are tuneable over a range of temperatures $\left(26-65^{\circ} \mathrm{C}\right)$ and pressures $(0-200 \mathrm{MPa})$.

Electrostatics clearly play a significant role in the large lattice parameters observed as noted by previous studies which have added the anionic lipids oleic acid, ${ }^{30}$ distearoyl phosphatidylglycerol (DSPG), ${ }^{20}$ dioleoyl phosphatidic acid (DOPA), ${ }^{31}$ DOPS $^{12,32}$ and DOPG $^{33}$ to MO. The largest lattice parameters reported previously were obtained in MO mixed with DOPS (225 $\AA$ ) and DSPG (268 $⿱$ A) in water. For MO : DOPA (90: $10 \mathrm{~mol} \%)$, lattice parameters of around $180 \AA$ have been obtained at $20^{\circ} \mathrm{C}$, however, addition of $1 \mathrm{M} \mathrm{NaCl}$ causes the lattice parameter to reduce significantly to around $100 \AA$ A. This highlights both the major effect of charge screening on very similar mixed lipid cubic systems to those studied here and the potential to use this in the future for control of swollen cubic structures.

Previous studies ${ }^{26,28,34}$ have demonstrated that increasing temperature tends to cause a decrease in the lattice parameter of bicontinuous cubic phases due to increased lipid chain conformational disorder leading to an increase in the magnitude of the monolayer spontaneous inverse curvature. Conversely, increasing pressure causes lipid chain ordering and so tends to lead to an increase in bicontinuous cubic lattice parameters. However, as seen in these investigations, the effect of temperature and pressure can be more complicated with the overall structural response of the system being determined by a delicate balance of forces.

The phase behaviour of hydrated monoolein has been well documented. ${ }^{25,35,36}$ At hydrations exceeding $45 \mathrm{wt} \%$, between 20 and $85{ }^{\circ} \mathrm{C}$, the system adopts the inverse bicontinuous cubic $P n 3 m$ phase coexisting with excess water. ${ }^{25}$ The lattice parameter of the $P n 3 m$ phase decreases with increasing temperature and increases with pressure as expected. ${ }^{34}$

Adding charged lipids to a bilayer can alter many of its structural and mechanical properties. ${ }^{37}$ Most uncharged lipid mesophases swell with increasing hydration up to a maximum lattice parameter and then plateau whereas bilayers composed solely of charged lipids can swell indefinitely. ${ }^{38,39}$ The samples in this study were hydrated to $70 \mathrm{wt} \%$ water, (the maximum water content that was found to give consistently intense, well resolved diffraction patterns). Although this is above the excess water point for pure $\mathrm{MO},{ }^{25}$ addition of charged lipids is likely to significantly increase the amount of water that the lipid structure can accommodate. The constant mean curvature structural model for bicontinuous cubic phases can be used to calculate the water volume fraction from the lattice parameter and bilayer thickness. ${ }^{28}$ We have calculated the composition of the highly swollen structures generated here (assuming the bilayer properties are the same as those for pure MO) to assess if the samples are in excess water (see ESI $\dagger$ for calculations). For a lattice parameter of $400 \AA$, we obtain a water content of 78.5 wt $\%$ for the $\operatorname{Im} 3 m$ cubic phase. This is almost $10 \mathrm{wt} \%$ higher than the amount of water added in the preparation stage; this discrepancy is likely to be due to the assumption that the 
molecular area and volume ${ }^{28,40}$ are equal to the parameters known for pure MO. However, importantly, these calculations imply that the samples may not be in excess water.

\section{MO : DOPG-DOPS binary mixtures}

Binary mixtures of MO with DOPG-DOPS show an increase in lattice parameter with an increase in temperature and only a small variation with pressure. This behaviour differs from that of pure MO and previously reported pressure effects on other single component cubic phases. ${ }^{34}$

The increased lattice parameter in both binary systems with increasing temperature implies that charged headgroup repulsion is dominating the bilayer behaviour, over-riding the increase in conformational disorder within the chain region due to the increased temperature. As these samples have been prepared in water, the electrostatic behaviour of the systems are dominated by the headgroup interactions of DOPG and DOPS.

DOPG and DOPS both have a single negatively charged headgroup with an associated sodium counterion. An increase in temperature is likely to promote dissociation of the counterion, leading to a greater headgroup-headgroup repulsion. ${ }^{41-43}$ This increased headgroup repulsion must outweigh the increase in conformational disorder in the chain region, resulting in an increased lattice parameter with increasing temperature.

The effects of pressure on charged lipids and counterion condensation are less well characterised. Increasing pressure increases conformational order in the chain region, which usually leads to an increase in lattice parameter in bicontinuous cubic phases. ${ }^{26,28}$ As the lattice parameter only varies by $4 \AA$ Aver $200 \mathrm{MPa}\left(\right.$ at $45{ }^{\circ} \mathrm{C}$ ) it seems likely that the effect of changes in counterion condensation due to increasing pressure are similar in magnitude but opposite to the effect of chain conformational ordering. This implies that pressure causes an increase in counterion condensation, which would lead to a reduction in the net headgroup charge, so a reduction in the apparent headgroup size and an increase in the magnitude of the monolayer spontaneous inverse curvature (counteracting the decrease caused by chain ordering). At $25{ }^{\circ} \mathrm{C}$, a decrease in the lattice parameter of $15 \AA$ is observed over the same pressure range, implying that the reduction in electrostatic repulsion is more dominant over the increased ordering in the chain region than at $45^{\circ} \mathrm{C}$.

\section{MO : chol : DOPG-DOPS ternary mixtures}

Ternary mixtures of MO : chol : DOPG show a decrease in lattice parameter both with increasing temperature and increasing pressure. The reduction in lattice parameter with increasing temperature (in contrast to the binary MO:DOPG mixture which shows an increase in lattice parameter with increasing temperature), indicates that the addition of cholesterol has a significant influence on the structural properties of the bilayer. An increased sensitivity to pressure was also noted at all temperatures, with a reduction in the lattice parameter of at least $20 \AA$ between atmospheric pressure and $200 \mathrm{MPa}$ for all temperatures studied.
Cholesterol incorporation into fluid bilayers generally leads to increased ordering in the hydrocarbon chains and an increase in lattice parameter. ${ }^{12,44,45}$ However, cholesterol also undergoes rapid axial motion which increases with increasing temperature and causes significant thermal motion in the hydrocarbon chain region. ${ }^{45}$ This additional thermal motion and conformational disorder in the chain region may contribute to the reduction in lattice parameter noted with increasing temperature. Additionally, theoretical studies have shown that while the hydrophobic backbone of cholesterol is inserted into the hydrocarbon region, contact is maintained between the cholesterol OH group and the lipid polar headgroup region ${ }^{46}$ and this may lead to a change in the lipidcounterion interaction. The modulation of the cubic structural behaviour by cholesterol is supported by the data in Fig. 4 which shows that the sample containing significantly less cholesterol shows a smaller reduction in lattice parameter with increasing temperature.

The effects of pressure on the cubic structure are more complex. Whilst the binary MO : DOPG mixtures show only a small change in lattice parameter with pressure, both ternary mixtures containing DOPG showed a significant decrease in lattice parameter with increasing pressure across all temperatures studied. Previous studies of the effect of hydrostatic pressure on lipid mixtures have demonstrated that cholesterol can reduce the susceptibility of the hydrocarbon chains to hydrostatic pressure induced ordering. ${ }^{47,48}$ This is consistent with an increase in pressure causing an increase in counterion condensation (as suggested previously), but with less change in the hydrocarbon chain ordering than in the non-cholesterol containing mixtures. Overall, the dominant effect of reduced headgroup repulsion leads to an increase in the magnitude of the spontaneous monolayer inverse curvature and so a reduction in lattice parameter with increasing pressure.

Ternary mixtures of MO : chol : DOPS show an increase in lattice parameter with increasing temperature and a decrease in lattice parameter with increasing pressure. The increase in lattice parameter with temperature corresponds to the changes observed in the binary mixtures, although it is different from the ternary mixture containing DOPG. The difference between the behaviour of the ternary mixtures is likely to be due to the difference in headgroup size and structure between DOPG and DOPS; DOPS has a larger headgroup than DOPG and so will exhibit greater steric repulsion in the headgroup region. This repulsion (and the difference in steric repulsion between DOPS and DOPG) is likely to increase with temperature (alongside the increased electrostatic repulsion), driving a flattening of the lipid monolayers and so an increase in the cubic lattice parameter. Whilst studies have shown that DOPS has a marginally higher counterion association than DOPG, ${ }^{37}$ the interplay between counterion association and cholesterol may influence the structural behaviour of the membrane and also affect the subsequent lattice parameter.

The reduction in lattice parameter with pressure in the DOPS ternary mixtures correlates well with the DOPG ternary mixtures. A larger decrease in lattice parameter is seen for lower cholesterol concentrations, supporting the previous idea that 
incorporation of cholesterol reduces the ordering effect of pressure in the hydrocarbon chain region.

Overall, the structures of highly swollen cubic phases have been found to be highly sensitive to temperature, pressure and composition, and are controlled by a complex interplay between electrostatic interactions, thermal motion and steric repulsion. By carefully varying temperature, pressure and composition we have demonstrated that the lattice parameters of these structures can be tuned over a wide size range from 300 to $480 \AA$.

\section{Conclusions}

This work has demonstrated the largest lipid based, protein free, bicontinuous cubic phases found to date. These structures approach the length scales only previously seen in vivo and we have been able to tune these by changing pressure, temperature and composition.

The sensitivity of these highly swollen mixtures to temperature, pressure and charged lipids will pave the way for the rational design of swollen cubic phases. These tuneable cubic structures will significantly enhance the pool of available structures for numerous techniques including in meso protein crystallisation, ${ }^{2,49}$ enzymatic studies, ${ }^{19}$ drug encapsulation and therapeutic delivery. ${ }^{6}$ By reaching new length scales and being able to demonstrate control of these structures we are removing the bottleneck which has been hampering these studies in the past which will lead to many exciting future developments.

\section{Acknowledgements}

This work was supported by EPSRC Platform grant EP/G00465X/ 1, EPSRC Programme grant EP/J017566/1 and by EPSRC Centre for Doctoral Training Studentships from the Institute of Chemical Biology EP/F500076/1 awarded to Edward Parsons and Nicola McCarthy. We acknowledge Diamond Light Source and the European Synchrotron Radiation Facility for provision of synchrotron beamtime and we would like to thank Dr Andy Smith for assistance using beamline I22 (DLS) and Dr Michael Sztucki for assistance using beamline ID02 (ESRF).

\section{Notes and references}

1 H. T. McMahon and J. L. Gallop, Nature, 2005, 438, 590-596.

2 M. Caffrey, D. Li, N. Howe and S. T. Shah, Philos. Trans. R. Soc. London, Ser. B, 2014, 369, 20130621.

3 V. Cherezov, J. Clogston, M. Z. Papiz and M. Caffrey, J. Mol. Biol., 2006, 357, 1605-1618.

4 V. Cherezov, Curr. Opin. Struct. Biol., 2011, 21, 559-566.

5 R. Negrini and R. Mezzenga, Langmuir, 2012, 28, 1645516462.

6 R. Negrini, A. Sanchez-Ferrer and R. Mezzenga, Langmuir, 2014, 30, 4280-4288.

7 J. M. Seddon and R. H. Templer, Handbook of Biological Physics, 1995, ch. 3, pp. 97-160.

8 Z. A. Almsherqi, S. D. Kohlwein and Y. Deng, J. Cell Biol., 2006, 173, 839-844.
9 Y. Deng, M. Marko, K. F. Buttle, A. Leith, M. Mieczkowski and C. A. Mannella, J. Struct. Biol., 1999, 127, 231-239.

10 C. Leal, N. F. Bouxsein, K. K. Ewert and C. R. Safinya, J. Am. Chem. Soc., 2010, 132, 16841-16847.

11 C. V. Kulkarni, O. Ces, R. H. Templer and J. M. Seddon, Soft Matter, 2013, 9, 6525.

12 V. Cherezov, J. Clogston, Y. Misquitta, W. Abdel-Gawad and M. Caffrey, Biophys. J., 2002, 83, 3393-3407.

13 R. Bruinsma, J. Phys., 1992, II, 425-451.

14 H. M. Nielsen, J. Thewalt, L. Miao, J. H. Ipsen, M. Bloom, M. J. Zuckermann and O. G. Mouritsen, Europhys. Lett., 2000, 52, 368-374.

15 P. Meleard, C. Gerbeaud, T. Pott, L. Fernandez-Puente, I. Bivas, M. D. Mitov, J. Dufourcq and P. Bothorel, Biophys. J., 1997, 72, 2616-2629.

16 M. Caffrey, Biochem. Soc. Trans., 2011, 39, 725-732.

17 A. Zabara and R. Mezzenga, Soft Matter, 2012, 8, 6535.

18 B. Angelov, A. Angelova, V. M. Garamus, G. Lebas, S. Lesieur, M. Ollivon, S. S. Funari, R. Willumeit and P. Couvreur, J. Am. Chem. Soc., 2007, 129, 13474-13479.

19 W. Sun, J. J. Vallooran, A. Zabara and R. Mezzenga, Nanoscale, 2014, 6, 6853-6859.

20 J. Engblom, Y. Miezis, T. Nylander, V. Razumas and K. Larsson, Prog. Colloid Polym. Sci., 2000, 116, 9-15.

21 V. Cherezov, D. P. Siegel, W. Shaw, S. W. Burgess and M. Caffrey, J. Membr. Biol., 2003, 195, 165-182.

22 D. P. Siegel and B. G. Tenchov, Biophys. J., 2008, 94, 39873995.

23 Y. La, C. Park, T. J. Shin, S. H. Joo, S. Kang and K. T. Kim, Nat. Chem., 2014, 6, 534-541.

24 N. J. Brooks, B. L. Gauthe, N. J. Terrill, S. E. Rogers, R. H. Templer, O. Ces and J. M. Seddon, Rev. Sci. Instrum., 2010, 81, 064103.

25 H. Qiu and M. Caffrey, Biomaterials, 2000, 21, 223-234.

26 N. J. Brooks, O. Ces, R. H. Templer and J. M. Seddon, Chem. Phys. Lipids, 2011, 164, 89-98.

27 F. Caboi, G. S. Amico, P. Pitzalis, M. Monduzzi, T. Nylander and K. Larsson, Chem. Phys. Lipids, 2001, 2001, 47-62.

28 T. Y. Tang, N. J. Brooks, C. Jeworrek, O. Ces, N. J. Terrill, R. Winter, R. H. Templer and J. M. Seddon, Langmuir, 2012, 28, 13018-13024.

29 J. M. Seddon, A. M. Squires, C. E. Conn, O. Ces, A. J. Heron, X. Mulet, G. C. Shearman and R. H. Templer, Philos. Trans. $R$. Soc. London, Ser. A, 2006, 364, 2635-2655.

30 Y. Aota-Nakano, S. Jie Li and M. Yamazaki, Biochim. Biophys. Acta, 1999, 1461, 96-102.

31 S. Jie Li, Y. Yamashita and M. Yamazaki, Biophys. J., 2001, 81, 983-993.

32 Y. Okamoto, S. M. Masum, H. Miyazawa and M. Yamazaki, Langmuir, 2008, 24, 3400-3406.

33 T. S. Awad, Y. Okamoto, S. M. Masum and M. Yamazaki, Langmuir, 2005, 21, 11556-11561.

34 C. Czeslik, R. Winter, G. Rapp and K. Bartels, Biophys. J., 1995, 68, 1423-1429.

35 J. Briggs, H. Chung and M. Caffrey, J. Phys., 1996, 6, 723-751. 36 E. S. Lutton, J. Am. Oil Chem. Soc., 1965, 42, 1068-1070. 
37 G. Lindblom, L. Rilfors, J. B. Hauksson, I. Brentel, M. Sjolund and B. Bergenstahl, Biochemistry, 1991, 30, 10938-10948.

38 S. C. Costigan, P. J. Booth and R. H. Templer, Biochim. Biophys. Acta, 2000, 1468, 41-54.

39 H. Hauser, Biochim. Biophys. Acta, Biomembr., 1984, 772, 3750.

40 G. C. Shearman, B. J. Khoo, M.-L. Motherwell, K. A. Brakke, O. Ces, C. Conn, J. M. Seddon and R. H. Templer, Langmuir, 2007, 23, 7276-7285.

41 D. Andelman, in Handbook of Biological Physics, ed. R. Lipowsky and E. Sackmann, Elsevier Science, 1995, vol. 1, ch. 12, pp. 603-641.

42 A. Lau and P. Pincus, Phys. Rev. E: Stat., Nonlinear, Soft Matter Phys., 2002, 66, 041501.
43 G. S. Manning, Q. Rev. Biophys., 1978, II, 179-246.

44 I. V. Polozov and K. Gawrisch, Biophys. J., 2006, 90, 20512061.

45 J. A. Clarke, J. M. Seddon and R. V. Law, Soft Matter, 2009, 5, 369.

46 A. Kessel, N. Ben-Tal and S. May, Biophys. J., 2001, 81, 643658.

47 O. Reis, R. Winter and T. W. Zerda, Biochim. Biophys. Acta, 1996, 1279, 5-16.

48 L. F. Braganza and D. L. Worcester, Biochemistry, 1986, 25, 7484-7488.

49 D. Li, S. T. Shah and M. Caffrey, Cryst. Growth Des., 2013, 13, 2846-2857. 